\title{
Phylogenetic study of H5 low pathogenic avian influenza viruses detected in wild birds in Poland in 2010-2015
}

\author{
Edyta Świętoń, Krzysztof Śmietanka \\ Department of Poultry Diseases, \\ National Veterinary Research Institute, 24-100 Pulawy, Poland \\ edyta.swieton@piwet.pulawy.pl
}

Received: June 23, 2017

Accepted: November 2, 2017

\begin{abstract}
Introduction: The genomes of nine H5 subtypes of low pathogenic avian influenza virus (LPAIV) strains identified in wild birds in Poland between 2010 and 2015 were sequenced, and their phylogenetic relationship was determined. Material and Methods: AIV genome segments were amplified by RT-PCR and the PCR products were sequenced using Sanger method. Phylogenetic trees were generated in MEGA6 software and digital genotyping approach was used to visualise the relationship between analysed strains and other AIVs. Results: High genetic diversity was found in the analysed strains as multiple subgroups were identified in phylogenetic trees. In the HA tree, Polish strains clustered in two distinct subclades. High diversity was found for PB2, PB1, PA and NP, since 5-8 sublineages could be distinguished. Each strain had a different gene constellation, although relationship of as much as six out of eight gene segments was observed between two isolates. A relationship with poultry isolates was found for at least one segment of each Polish strain. Conclusion: The genome configuration of tested strains indicates extensive reassortment, although the preference for specific gene constellation could be noticed. A significant relationship with isolates of poultry origin underlines the need for constant monitoring of the AIV gene pool circulating in the natural reservoir.
\end{abstract}

Keywords: birds, avian influenza virus, H5 subtype, phylogenetic analysis, Poland.

\section{Introduction}

Avian influenza virus (AIV) is one of the most important avian pathogens affecting a wide range of bird species. Wild aquatic birds, mostly the Anseriformes and Charadriiformes orders, are considered to be the natural reservoir for low pathogenic avian influenza viruses (LPAIV) and they can be a source of infection in poultry $(14,17)$. AIV classification is based on the combination of two surface proteins present in the virus particle haemagglutinin (HA) and neuraminidase (NA), which are divided into 16 and 9 subtypes, respectively. The AIV genome consists of eight negative-sense RNA segments, which enables reassortment of genes in case of co-infection of one cell with different AI viruses (24). Apart from the above-mentioned HA and
NA segments, AIV genome includes gene segments coding polymerase complex subunits (PB2, PB1, PA), nucleoprotein (NP), matrix and ion channel proteins $(\mathrm{M})$, and nonstructural and nuclear export proteins (NS). Additionally, translation from alternative ORFs or ribosomal frame shifting leads to generation of proteins influencing virus virulence, such as PB1-F2 and PA-X (28). The pathogenicity of AIV is primarily associated with the sequence of amino acids at the cleavage site of the HA protein. The LPAIV may acquire high virulence by mutation leading to an introduction of multiple basic amino acids (mainly arginine and lysine) into the HA cleavage site, which have so far been observed only for $\mathrm{H} 5$ and $\mathrm{H} 7$ subtypes (18). Therefore, in case of LPAI H5 and H7 outbreaks, there is a risk that the virus evolves into highly pathogenic form causing high mortality in 
infected flocks (1). Transmission of HPAIV back to migratory birds can result in spreading the virus over large areas (9). Furthermore, co-circulation of HPAIV and LPAIV in the same hosts is an opportunity for genome reshuffling in the reassortment process (8).

There have been several cases of LPAI and HPAI outbreaks in poultry in Europe $(4,6,7,12)$ that were induced by viruses which probably emerged from low pathogenic precursors of wild-bird origin $(5,11,13$, 22). The dynamic nature of AIV evolution is a challenge for diagnosis and control. For this reason, monitoring and characterisation of $\mathrm{H} 5$ and $\mathrm{H} 7$ AIVs in wild birds to follow the interactions in the pool of circulating viruses and emergence of novel molecular traits and genotypes is of great importance.

Surveillance for AIV in wild birds carried out in Poland in 2008-2015 showed a high diversity of subtypes with a substantial subset of H5 (26). In the present study, nine wild bird-origin H5 LPAIVs detected in Poland in 2010-2015 (thus prior to the emergence of H5N8 HPAIV in wild birds) were subjected to analysis. Partial or full sequences of all gene segments were obtained and their phylogenetic relationship was characterised to determine the diversity of gene lineages circulating in Poland and their association with other strains of wild bird and poultry origin.

\section{Material and Methods}

Viruses. Nine H5 LPAIVs detected in wild birds in Poland in 2010-2015 were used in the study (Table 1, Fig. 1). Six isolates were identified as H5N3, one as $\mathrm{H} 5 \mathrm{~N} 2$, one as H5N6, and one as H5N1. The viruses were detected in mallards $(6 \times)$, common teals $(1 \times)$, mute swans $(1 \times)$, and greylag goose $(1 \times)$. Five viruses were successfully isolated in SPF embryonated chicken eggs and infectious allantoic fluid was used for RNA extraction. In the case of negative isolation, RNAs extracted from cloacal swabs were used for further analysis. Additionally, H5 gene detected in a sample from a common teal, in which mixed infection was found ( $\mathrm{H} 5, \mathrm{H} 6, \mathrm{~N} 3$, and $\mathrm{N} 8$ genes were detected), was included in the HA gene analysis.

RNA extraction, RT-PCR, and sequencing. The RNA was extracted from $200 \mu \mathrm{L}$ of allantoic fluid or swab sample using RNeasy Mini kit (Qiagen, Germany) according to the manufacturer's instruction. Fragments of all gene segments were obtained in single or overlapping RT-PCR reactions using One Step RT-PCR kit (Qiagen) and specific primers designed by the authors (sequences and cycling conditions available upon request). Nucleotide sequences of the PCR products were determined using BigDye Terminator v3.1 Cycle Sequencing kit (Applied Biosystems, USA) according to the manufacturer's instruction. Capillary electrophoresis of the sequencing reaction products was performed in
3500 Genetic Analyzer (Applied Biosystems). Sequences were assembled and analysed with SeqMan, DNAStar LaserGene (version 8.1.3).

Phylogenetic analysis. Phylogenetic studies were based on the following regions of viral segments: HA: 57-1648, N1: 57-740, N2: 22-1410, N3: 301-704, PB1: 71-2078, PB2: 11-2228, PA: 261611, NS: 73-754, NP: 51-1412, M: 284-763. In the case of $\mathrm{A} / \mathrm{common}$ teal $/ 84 / 13$, due to the mixed infection and failure in isolating the virus in SPF embryonated eggs, only HA gene was taken into account. The determined sequences were aligned with selected sequences available from GenBank and EpiFLUTM database using ClustalW programme within MEGA 6 software (27). Phylogenetic trees were inferred with MEGA 6 software using the neighbour-joining method with 1,000 bootstrap replicates and the Kimura 2-parameter method for computing evolutionary distances. The trees were analysed to identify the relationship between Polish strains and other co-circulating $\mathrm{H} 5$ and non- $\mathrm{H} 5$ viruses for each gene segment. To visualise the association between genotypes and facilitate the analysis, the digital genotyping technique described by Nagy et al. (15) was used. Briefly, subgroups with high reliability supported by the bootstrap value of $\geq 90 \%$ were identified in each phylogenetic tree and a pixel array representing genotypes of selected strains was constructed. The presence of a coloured square at the intersection of two strains means that their genes belong to the same subgroup and the colour indicates the nucleotide similarity.

Gene sequences were submitted to the EpiFLUTM GISAID nucleotide sequence database (platform. gisaid.org) with the following isolate identification numbers: EPI ISL 234327, EPI ISL 234400 to EPI_ISL_23440 $\overline{4}, \overline{\text { EPI_ISL_} 23440 \overline{6}}$ to EPI_ISL $234 \overline{4} 08$, and EPI_ISL_266853.

\section{Results}

The phylogenetic trees constructed for all genome segments of Polish H5 strains showed their affiliation to the Eurasian lineage. High diversity was identified as multiple subclades within individual segments and various gene constellations could be observed. To illustrate the relationship of the analysed strains with each other and other isolates of wild bird and poultry origin, a digital genotyping approach was used with a set of sequences with observed relationship with Polish strains for at least two genes (Table 2, Fig. 3).

Phylogenetic analysis of the HA gene revealed that most of the analysed strains clustered within two subclades (Fig. 2). The first group comprised six Polish viruses, detected between 2013 and 2015, and European $\mathrm{H} 5 \mathrm{~N} 2$ and $\mathrm{H} 5 \mathrm{~N} 3$ isolates. The nucleotide sequence homology within this cluster ranged from 
$97.6 \%$ to $99.5 \%$. Polish strains $74 / 10,175 / 11$, and $80 / 12$ formed the second subgroup with H5N2 strains isolated from poultry in Italy and from a goose in the Netherlands. The strongest sequence identity was observed between $175 / 11$ and NL/2/10(H5N2) (99.7\%), nevertheless, all Polish strains showed high similarity $(98.8 \%-99.6 \%)$ with an Italian isolate from a guinea fowl.

In the case of NA, fragments of N2, N3, and N1 genes were examined. The N2 sequence of 74/10 clustered in a subgroup with gf/It/10(H5N2) (sequence homology 99.6\%) and other three wild-bird isolates, of which IC/1110/11(H5N2) and $\mathrm{NL} / 2 / 10(\mathrm{H} 5 \mathrm{~N} 2)$ were also present in HA tree in the first and second subgroup, respectively. The N1 gene of 141/15 showed the highest relationship with an H5N1 isolate from chicken in Scotland (98.6\%). The phylogeny of N3 revealed a separation of Polish strains into three subclades. One of them encompassed four Polish strains from 2013-2014 and the remaining two strains were located in different subclades.

The phylogenetic trees for PB2, PB1, PA, and NP genes showed a high level of genetic variability with clearly distinguishable subgroups. In the case of PB2, the analysed strains were grouped into five subclades. Interestingly, group 4 comprised the same set of Polish strains as group 1 in the HA phylogeny, but this subset was split in trees for the remaining internal genes. The PB1 tree could be divided into eight sublineages, and, except for $96 / 13$ and 76/13, each strain was located in a different subgroup. Slightly lower diversity was detected in PA and NP phylogenetic trees, since seven subclades in each were identified. In the case of NS and M segments, separation into subgroups was less pronounced with 1-3 branches with the bootstrap value exceeding $90 \%$. The topology of NS tree showed that the analysed strains belonged to both allele $\mathrm{A}$ and $\mathrm{B}$. Strains 76/13, 86-1/13, and 96/13 were grouped in one subclade within allele A, whereas 266/10, 10/14, and $141 / 15$ could not be assigned to any discrete subgroup. Allele B was represented by 74/10, 175/11, and $80 / 12$.

Trees for internal segments showed a complicated network of relationship between Polish strains. Additionally, numerous connections with poultry isolates were observed. The digital genotyping method demonstrated that there were no strains with identical gene configuration among the Polish isolates, whereas significant interchange of segments was observed (Fig. 3). Polish strains could be divided into two groups based on the genome constellation. The "old" group comprised 74/10, 266/10, 175/11, and $80 / 12$ and the "new" group gathered $76 / 13$, $86-1 / 13,96 / 13,10 / 14$, and 141/15. Members of the "old" group showed some level of uniqueness since 74/10, $175 / 11$, and $80 / 12$ shared only the HA gene, and no interrelationship was detected between them and $266 / 10$. However, other segments were closely related to European H5 and non-H5 isolates of wild bird and poultry origin. The highest overlap was observed between $74 / 10$ and NL/2/10(H5N2) - an H5N2 isolate from a greater white-fronted goose collected two months earlier in the Netherlands, which shared seven segments almost identical at the nucleotide level. Only the NS segments could not be assigned to one subgroup, but both belonged to allele B and showed high nucleotide homology $(99.7 \%)$. A portion of $74 / 10$-like genes was also detected in Italian H5N2 isolate from a guinea fowl, whereas 266/10-like genes were the least represented in the matrix.

Members of the "new" group showed much more connections with each other and recent poultry isolates. All of newer Polish isolates shared the PB2-HA constellation, and the isolates of $\mathrm{H} 5 \mathrm{~N} 3$ subtype also possessed highly similar NA segment. Additionally, isolates from 2013 shared NS segment, and $76 / 13$ and $96 / 13$ also shared PB1 and PA. No such PB2-HA-NA configuration could be observed in any recent European $\mathrm{H} 5 \mathrm{~N} 3$ strains, but the HA gene was most closely related to H5N2 isolate IC/1110/11, linking the "old" and "new" group, as its PB2, PB1, NP, and NA-like genes were detected in $80 / 12$, 266/10, 175/11, and 74/10, respectively. The remaining genes were dispersed throughout the plot to a varying extent. PB2 gene seemed to be particularly interesting, since similar sequences were also found in two $\mathrm{H} 7 \mathrm{~N} 7$ poultry isolates and $\mathrm{H} 10 \mathrm{~N} 7$ isolates from seals, and for some isolates these relationships also involved other segments. For example, a constellation of PB2-NP genes was found in 76/13, ch/NL/15 (H7N7) and sl/Ger/14(H10N7), or PB2-PA in 86-1/13 and ty/Ger/13(H7N7). Some gene overlap with H5N1 isolates from poultry was also observed, as 141/15like PA-NP-NA triple was retained in LPAIV $\mathrm{ch} / \mathrm{Sc} / 16(\mathrm{H} 5 \mathrm{~N} 1)$. Although not included in the digital genotyping matrix, a few cases of single-gene relationship with isolates of poultry origin were demonstrated. Similar PB1 was found in 10/14 and HPAIV H5N1 isolates from France. The PB1 gene of $175 / 11$ and the NP of $80 / 12$ were related with corresponding genes of $\mathrm{H} 7 \mathrm{~N} 7$ isolates from the Italian epidemic in 2013. The same genes (PB1 and NP) of $10 / 14$ and 86-1/13, respectively, were shared with chicken $\mathrm{H} 7 \mathrm{~N} 7$ isolate from England. Interestingly, a relationship with recent $\mathrm{H} 5 \mathrm{~N} 8 \mathrm{HPAI}$ isolates was observed for two Polish strains. In case of 141/15, the PB1 gene was grouped in one cluster with Polish and Czech H5N8 isolates from poultry, whereas the NP gene of $96 / 13$ was located in one subgroup with another H5N8 HPAIVs from Asia and Europe. 
Table 1. Characterisation of analysed H5 strains

\begin{tabular}{lllll}
\hline Virus & Abbreviated name & Location & Collection date & Subtype \\
\hline A/graylag goose/Poland/74/2010 & $74 / 10$ & Świnoujście & 25-03-2010 & H5N2 \\
A/mallard/Poland/266/2010 & $266 / 10$ & $\begin{array}{l}\text { Nowy Dwór } \\
\text { Mazowiecki }\end{array}$ & 22-10-2010 & H5N6 \\
A/mallard/Poland/175/2011 & $175 / 11$ & Żyrardów & $18-08-2011$ & H5N3 \\
A/common teal/Poland/80/2012 & $80 / 12$ & Jeziorsko & 02-09-2012 & H5N3 \\
A/mallard/Poland/76/2013 & $76 / 13$ & Szczecinek & $28-08-2013$ & H5N3 \\
A/common teal/Poland/84/2013* & $84 / 13$ & Jeziorsko & $28-08-2013$ & mixed \\
A/mute swan/Poland/86-1/2013 & $86-1 / 13$ & Ustków & H5N3 \\
A/mallard/Poland/96/2013 & 96/13 & Gdynia & Hog-2013 & H5N3 \\
A/mallard/Poland/10/2014 & $10 / 14$ & Olsztyn & $18-01-2014$ & H5N3 \\
\hline
\end{tabular}

* only H5 gene was used in the phylogenetic analysis

Table 2. Isolates used in the digital genotyping matrix

\begin{tabular}{|c|c|c|c|}
\hline Isolate name & Abbreviated name & Collection date & Isolate ID (EpiFlu) \\
\hline $\mathrm{A} /$ greater white-fronted goose/Netherlands/2/2010(H5N2) & $\mathrm{NL} / 2 / 10(\mathrm{H} 5 \mathrm{~N} 2)$ & 29-01-2010 & EPI_ISL_243542 \\
\hline A/great black-backed gull/Iceland/1110/2011(H5N2) & $\mathrm{IC} / 1110 / 11(\mathrm{H} 5 \mathrm{~N} 2)$ & $16-10-2011$ & EPI_ISL_148203 \\
\hline A/guinea fowl/Italy/10VIR-218-134/2010(H5N2) & $\mathrm{gf} / \mathrm{It} / 10(\mathrm{H} 5 \mathrm{~N} 2)$ & $12-01-2010$ & EPI_ISL_144902 \\
\hline A/goose/Germany/R3160/09(H5N2) & gs/Ger/09(H5N2) & $12-2009$ & EPI_ISL_70400 \\
\hline A/turkey/Germany-NI/R534/2013(H7N7) & $\mathrm{ty} / \mathrm{Ger} / 13(\mathrm{H} 7 \mathrm{~N} 7)$ & 11-04-2013 & EPI_ISL_146472 \\
\hline $\begin{array}{l}\text { A/chicken/NL-Barneveld/15004745-006- } \\
\text { 010/2015(H7N7) }\end{array}$ & $\mathrm{ch} / \mathrm{NL} / 15(\mathrm{H} 7 \mathrm{~N} 7)$ & $11-03-2015$ & EPI_ISL_192666 \\
\hline A/harbour seal/Germany/1/2014(H10N7) & $\mathrm{sl} / \mathrm{Ger} / 14(\mathrm{H} 10 \mathrm{~N} 7)$ & $07-10-2014$ & EPI_ISL_170566 \\
\hline $\mathrm{A} /$ chicken/Scotland/532/2016(H5N1) & $\mathrm{ch} / \mathrm{Sc} / 16(\mathrm{H} 5 \mathrm{~N} 1)$ & 09-01-2016 & EPI_ISL_205833 \\
\hline
\end{tabular}




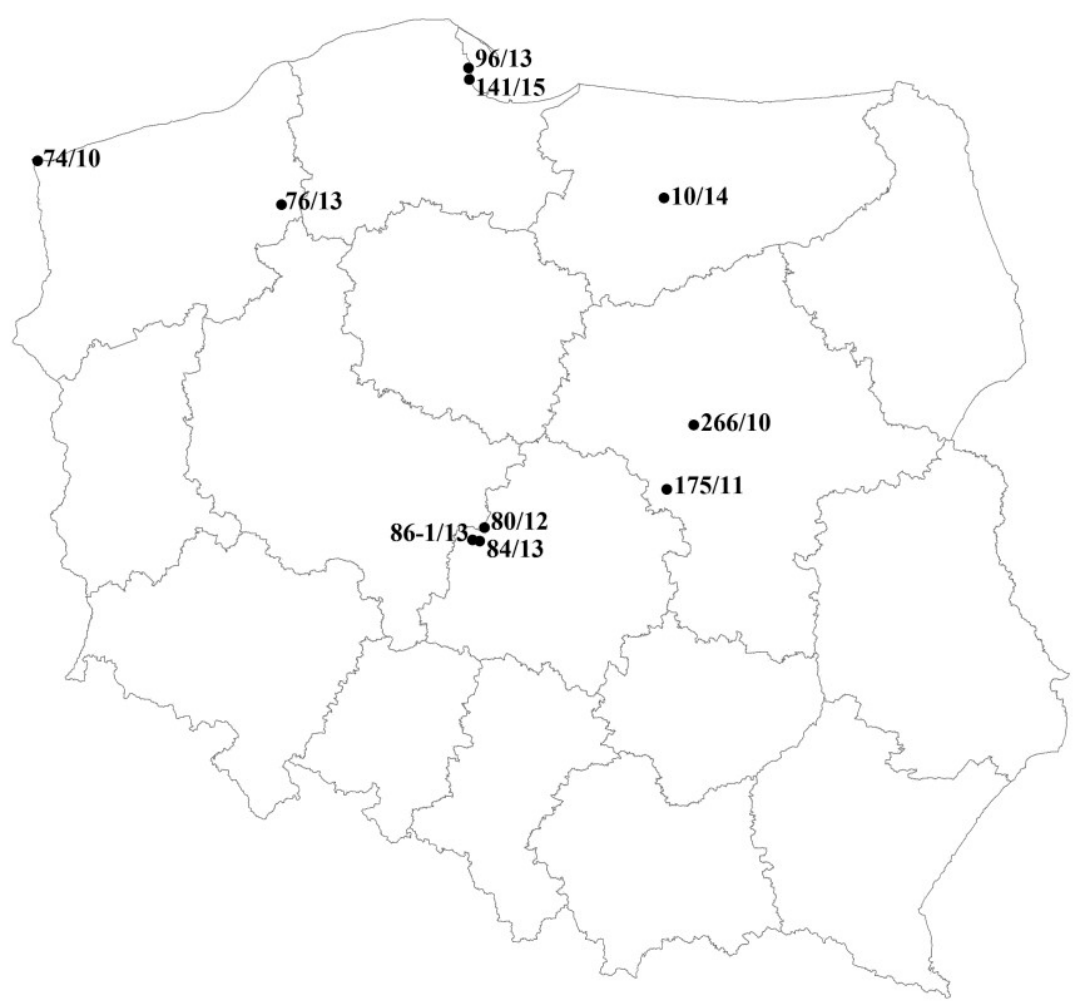

Fig. 1. The location of wild birds from which samples positive for H5 LPAIV were collected

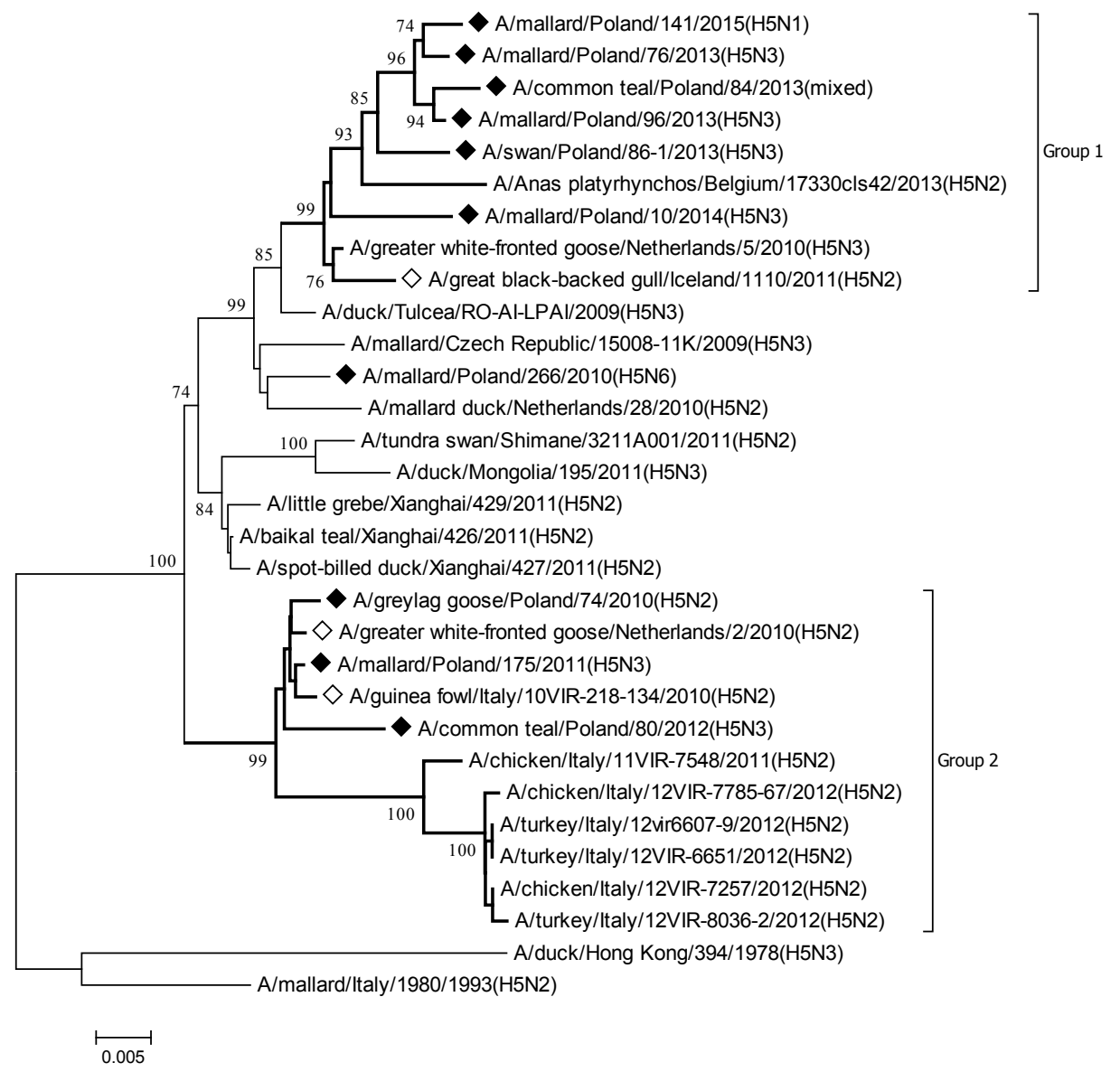

Fig. 2. Phylogenetic tree for $\mathrm{H} 5$ gene. Strains sequenced in the present study are indicated with a black diamond, and strains used for comparison in digital genotyping matrix with a white diamond. Bootstrap values of $\geq 70 \%$ are shown next to the branches 


\begin{tabular}{|c|c|c|c|c|c|c|c|c|c|c|c|c|c|c|c|c|c|c|c|c|c|c|c|c|c|c|}
\hline Isolate ID & \multicolumn{5}{|c|}{$74 / 10$} & \multicolumn{6}{|c|}{$266 / 10$} & \multicolumn{7}{|c|}{$175 / 11$} & \multicolumn{7}{|c|}{$80 / 12$} & \\
\hline Segment number & \begin{tabular}{l|l|}
1 & 2 \\
\end{tabular} & \begin{tabular}{|l|l|}
3 & 4 \\
\end{tabular} & \begin{tabular}{|l|l|}
5 & 6 \\
\end{tabular} & 7 & 8 & \begin{tabular}{|l|l}
1 & 2 \\
\end{tabular} & \begin{tabular}{l|l}
2 & 3 \\
\end{tabular} & 4 & \begin{tabular}{|l|l|l|}
5 & 6 \\
\end{tabular} & \begin{tabular}{l|l}
6 & 7 \\
\end{tabular} & \begin{tabular}{l|l|}
7 & 8 \\
\end{tabular} & 1 & 2 & 3 & \begin{tabular}{l|l}
4 & 5 \\
\end{tabular} & 56 & 7 & 8 & 1 & 2 & \begin{tabular}{|l|l}
3 & 4 \\
\end{tabular} & \begin{tabular}{|l|l|}
4 & 5 \\
\end{tabular} & \begin{tabular}{|l|l|}
6 \\
\end{tabular} & 7 & 8 & \\
\hline $74 / 10$ & & & & & & & & & & & & & & & & & & & & & & & & & & \\
\hline $266 / 10$ & & & & & & & & & & & & & & & & & & & & & & & & & & \\
\hline $175 / 11$ & & & & & & & & & & & & & & & & & & & & & & & & D & 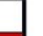 & \\
\hline $80 / 12$ & & & & & & & & & & & & & & & & & & & & & & & & & & \\
\hline $76 / 13$ & & & & & & & & & & & & & & & & & & & & & & & & & & Nucleotide \\
\hline $86-1 / 13$ & & & & & & & & & & & & & & & & & & & & & & & & & & homology \\
\hline $96 / 13$ & & & & & & & & & & & & & & & & & & & & & & & & & & $99,6-100 \%$ \\
\hline $10 / 14$ & & & & & & & & & & & & & & & & & & & & & & & & & & $99,1-99,5 \%$ \\
\hline $141 / 15$ & & & & & & & & & & & & & & & & & & & & & & & & & & $99,1-99,5 \%$ \\
\hline $\mathrm{NL} / 2 / 10(\mathrm{H} 5 \mathrm{~N} 2)$ & & & & & & & & & & & & & & & & & & & & & & & & & & $98,6-99,0 \%$ \\
\hline $\mathrm{IC} / 1110 / 11(\mathrm{H} 5 \mathrm{~N} 2)$ & & & & & & & & & & & & & & & & & & & & & & & & & & $98,1-98,5 \%$ \\
\hline $\mathrm{gf} / \mathrm{It} / 10(\mathrm{H} 5 \mathrm{~N} 2)$ & & & & & & & & & & & & & & & & & & & & & & & & & & $97,6-98,0 \%$ \\
\hline gs/Ger/09(H5N2) & & & & & & & & & & & & & & & & & & & & & & & & & & \\
\hline tv/Ger/13(H7N7) & & & & & & & & & & & & & & & & & & & & & & & & & & \\
\hline $\mathrm{ch} / \mathrm{NL} / 15(\mathrm{H} 7 \mathrm{~N} 7)$ & & & & & & & & & & & & & & & & & & & & & & & & & & \\
\hline sl/Ger/14(H10N7) & & & & & & & & & & & & & & & & & & & & & & & & & & \\
\hline $\mathrm{ch} / \mathrm{Sc} / 16(\mathrm{H} 5 \mathrm{~N} 1)$ & & & & & & & & & & & & & & & & & & & & & & & & & & \\
\hline
\end{tabular}

\begin{tabular}{|c|c|c|c|c|c|c|c|c|c|c|c|c|c|c|c|c|c|c|c|c|c|c|c|c|c|c|c|c|c|c|c|c|c|}
\hline Isolate ID & \multicolumn{6}{|c|}{$76 / 13$} & \multicolumn{7}{|c|}{$86-1 / 13$} & \multicolumn{7}{|c|}{$96 / 13$} & \multicolumn{6}{|c|}{$10 / 14$} & \multicolumn{7}{|c|}{$141 / 15$} \\
\hline Segment number & 122 & 3 & 4 & \begin{tabular}{l|l}
5 & $t$ \\
\end{tabular} & 67 & $\begin{array}{l}7 \\
\end{array}$ & 1 & 2 & \begin{tabular}{|l|l|l|}
3 & \\
\end{tabular} & \begin{tabular}{l|l}
4 & 5 \\
\end{tabular} & 6 & 7 & 8 & 12 & 2 & \begin{tabular}{|l|l}
3 & 4 \\
\end{tabular} & 5 & 6 & 7 & 8 & $1 \quad 2$ & 3 & 4 & \begin{tabular}{|l|l}
5 & \\
\end{tabular} & \begin{tabular}{l|l}
6 & 7 \\
\end{tabular} & 8 & 1 & 2 & \begin{tabular}{|l|l}
3 & 4 \\
\end{tabular} & & 6 & & 8 \\
\hline $74 / 10$ & & & & & & & & & & & & & & & & & & & & & & & & & & & & & & & & & \\
\hline $266 / 10$ & & & & & & & & & & & & & & & & & & & & & & & & & & & & & & & & & \\
\hline $175 / 11$ & & & & & & & & & & & & & & & & & & & & & & & & & & & & & & & & & \\
\hline $80 / 12$ & & & & & & & & & & & & & & & & & & & & & & & & & & & & & & & & & \\
\hline $76 / 13$ & & & & & & & & & & & & & & & & & & & & & & & & & & & & & & & & & \\
\hline $86-1 / 13$ & & & & & & & & & & & & & & & & & & & & & & & & & & & & & & & & & \\
\hline $96 / 13$ & & & & & & & & & & & & & & & & & & & & & & & & & & & & & & & & & \\
\hline $10 / 14$ & & & & & & & & & & & & & & & & & & & & & & & & & & & & & & & & & \\
\hline $141 / 15$ & & & & & & & & & & & & & & & & & & & & & & & & & & & & & & & & & \\
\hline $\mathrm{NL} / 2 / 10(\mathrm{H} 5 \mathrm{~N} 2)$ & & & & & & & & & & & & & & & & & & & & & & & & & & & & & & & & & \\
\hline $\mathrm{IC} / 1110 / 11(\mathrm{H} 5 \mathrm{~N} 2)$ & & & & & & & & & & & & & & & & & & & & & & & & & & & & & & & & & \\
\hline $\mathrm{gf} / \mathrm{It} / 10(\mathrm{H} 5 \mathrm{~N} 2)$ & & & & & & & & & & & & & & & & & & & & & & & & & & & & & & & & & \\
\hline gs/Ger/09(H5N2) & & & & & & & & & & & & & & & & & & & & & & & & & & & & & & & & & \\
\hline ty/Ger/13(H7N7) & & & & & & & & & & & & & & & & & & & & & & & & & & & & & & & & & \\
\hline $\mathrm{ch} / \mathrm{NL} / 15(\mathrm{H} 7 \mathrm{~N} 7)$ & & & & & & & & & & & & & & & & & & & & & & & & & & & & & & & & & \\
\hline sl/Ger/14(H10N7) & & & & & & & & & & & & & & & & & & & & & & & & & & & & & & & & & \\
\hline $\mathrm{ch} / \mathrm{Sc} / 16(\mathrm{H} 5 \mathrm{~N} 1)$ & & & & & & & & & & & & & & & & & & & & & & & & & & & & & & & & & \\
\hline
\end{tabular}

Fig. 3. Digital genotyping matrix generated for the analysed H5 strains and selected isolates from Genbank and EpiFlu databases. Coloured pixel at the intersection of two strains indicates they were located in the same subgroup in the phylogenetic tree for the given genome segment. The colour shows the range of nucleotide homology as demonstrated in the legend on the right. The segment numbering complies with standard numbering according to segment length (1-PB2, 2-PB1, 3-PA, 4-HA, 5-NP, 6-NA, 7-M, 8-NS). Empty fields mean lack of relationship

\section{Discussion}

The diversity of AIVs circulating in wild birds is a result of various subtype combinations and is further enriched by a variety of internal gene lineages. The exchange of genome segments between different AIVs in reassortment process contributes to the maintenance of diversity and can result in emergence of viruses with new biological properties. Occasionally, viruses are transmitted from the wild bird reservoir to poultry which, in the case of H5 and H7 subtypes, can have serious clinical and economic effect. In Poland, outbreaks caused by LPAI H5 and $\mathrm{H} 7$ viruses have been rare, with the most recent documented in 1990s (25). However, the presence of seroreagents in duck and geese flocks reared in free range systems suggests the occurrence of asymptomatic infections caused by LPAI viruses of wild bird origin (19). Furthermore, a few recent cases of LPAI outbreaks in poultry recorded in Europe have been caused by viruses derived from wild birds (10,
12, 20). Studies characterising the viruses from both wild birds and poultry have shown constant gene flow between various subtypes $(4,15,23)$. Molecular and phylogenetic analysis of French H5 strains isolated between 2002 and 2008 from domestic ducks showed high complexity of identified genotypes and presence of uncommon molecular features, such as insertions in PB1 and NS1, and truncation of PB1-F2 protein (4). Similarly, high heterogeneity of gene pool of H5 AIV isolates from wild birds was demonstrated in Nigeria, South Korea, and Japan (2, 21, 23). Gene exchange between Eurasian, North American, and African lineages was also observed for some isolates $(2,21)$. The threat from LPAIV is not the disease itself, but its propensity to become highly pathogenic when passaged in gallinaceous poultry. Therefore, characterisation of notifiable subtypes of AIV circulating in wild birds complements the monitoring programmes and gives insight into the interplay between various subtypes and strains of the same subtype. 
Phylogenetic analysis of all eight gene segments of H5 strains collected in Poland during five years showed that all of them belonged to the Eurasian lineage. However, high genetic diversity was observed, indicated by a relationship with a spectrum of genes circulating in Europe in various subtype combinations, suggesting that the genotypes of Polish strains are the effect of many reassortment events. Phylogenetic trees generated for each genome segment showed different levels of heterogeneity for individual genes. In the case of HA genes, two main subgroups comprising Polish strains could be distinguished, reflecting separation from the temporal (but not geographical) point of view. On the other hand, phylogenetic trees for PB2, PB1, $\mathrm{PA}$, and NP genes showed more diversity as at least five distinct subclades were identified in each. A different situation was observed for NS and M segments, in which separation into subgroups was not well established, except for NS division into alleles A and B. Regarding NA segment, four different subtypes were identified, with the majority of strains classified as N3, and single strains belonging to N1, N2, and N6.

To organise the data obtained from phylogenetic trees and collate them in an intelligible form, the digital genotyping method was applied enabling easy analysis of genome constellations of individual strains (15). Colouring applied in our study, which depends on the nucleotide similarity, simultaneously gives insight into the gene homology. The pixel pattern in the digital genotyping matrix indicated various gene overlap between Polish strains, and showed that each of them possessed a different gene constellation. Genes of Polish isolates could be divided into two pools - "old" represented by isolates detected in 2010-2012, and "new" consisting of strains from 2013-2015. The intragroup gene overlap in the "old" group was low, and so was the inter-group interchange of segments. On the other hand, the pool of viruses detected in 2013-2015 shared at least a constellation of two genes (PB2, HA), but a relationship of as much as six genes (PB2, PB1, PA, HA, NA, NS) was observed for $76 / 13$ and 96/13, two isolates detected two months apart at a distance of approximately $150 \mathrm{~km}$. A significant gene overlap (PB2, HA, NA, NS) was also found between these two strains and 86-1/13 identified in the same time interval, but in a more distant location. The strain 10/14 (detected three months later) possessed a subset of PB2, HA, and NA, and 141/15 collected more than two years later in the same location as $96 / 13$ retained the PB2-HA constellation. Furthermore, the N3 gene of isolates detected in 2013-2014 showed a significant distinctiveness from non-Polish sequences, as the closest relatives were $\mathrm{H} 4 \mathrm{~N} 3$ and $\mathrm{H} 2 \mathrm{~N} 3$ isolates from mallards isolated in 2009 and 2010, respectively. This may suggest that Polish 2013-2014-year H5N3 isolates are a "closed circle", in which genes such as NP underwent complete rearrangement, but PB2-HA gene constellation was preserved for three years, which may indicate their increased compatibility. An extensive reassortment of AIVs at a local level over short time was demonstrated $(16,29)$. On the other hand, circulation of specific gene constellation without reassortment for a prolonged time was also observed $(4,15)$, to which the existence of frozen evolution of AIV may contribute as a result of persistence in the environment without replication (16). The case of 84/10 represents an example of an opportunity for reassortment. The common teal was found to be coinfected with two AIV subtypes as H5, H6, N3, and N8 genes were identified. Although the exact subtype combination could not be determined, full sequence of H5 segment was obtained, which clustered with other isolates detected in 2013-2015.

In contrast, isolates collected between 2010 and 2012 showed more partitioning as the HA gene was the only linkage between $74 / 10,175 / 11$, and $80 / 12$, whereas 266/10 showed no relationship with any of these isolates. Detection of similar HA gene in three isolates within three years and lack of relationship between remaining genes indicates they had a common ancestor, but excessive reassortment resulted in a complete exchange of genes other than HA. Almost full homology of genes of 74/10 and NL/2/10(H5N2) and a significant subset of similar genes detected in $\mathrm{gf} / \mathrm{It} / 10(\mathrm{H} 5 \mathrm{~N} 2)$ indicates they all share a very recent common ancestor. Both $\mathrm{NL} / 2 / 10(\mathrm{H} 5 \mathrm{~N} 2)$ and gf/It/10(H5N2) were identified about two months earlier than 74/10, suggesting that NL/2/10(H5N2)-like virus was a donor of four genes to $\mathrm{gf} / \mathrm{It} / 10$ (H5N2)-like virus, but reached Poland in an almost unchanged form.

A relationship with poultry isolates was also observed for other genes of Polish strains. Isolates $175 / 11,74 / 10$, and 86-1/13 shared a few genes with a German H5N2 isolate from a domestic goose. The PB2 of isolates $76 / 13,86-1 / 13,96 / 13,10 / 14$, and 141/15 showed high homology with PB2 of LPAI H7N7 virus from poultry and H10N7 virus detected in recent years in seals. The 141/15-like constellation of PA, NP, and NA was detected in Scottish H5N1 LPAIV, whereas PB1 segment of 10/14 showed a high relationship with the French $\mathrm{H} 5 \mathrm{~N} 1$, a highly pathogenic virus derived from low pathogenic progenitor as a result of mutation accumulation (5). Similar results providing evidence of virus spill-over from wild birds to poultry and constant gene flow between different viruses were obtained by other authors as well $(4,15$, 23). However, crossing the species barrier by AIV is not restricted only to wild bird-poultry transmission, as AIVs have also proven to be able to transmit and adapt to mammals. The above-mentioned H10N7 virus, which has caused mass mortality among harbour seals in Sweden, Denmark, Germany, and the Netherlands since spring 2014, was shown to originate from wild birds (3).

Although wild birds are a natural reservoir for low pathogenic AIV, numerous cases of HPAIV H5Nx in wild birds have been recently recorded, indicating they can be a vector carrying the virus over large distances 
(9). Co-infection of wild birds with HPAIV and LPAIV creates opportunities for reassortment and emergence of new genotypes. Relationship of internal genes of two Polish strains with reassortant H5N8 HPAIVs of clade 2.3.4.4 is evidence of this phenomenon. However, the spatial and temporal characteristics of this gene exchange remains to be investigated.

Summarising, the present study shows a complicated network of interrelationship between H5 LPAI viruses occurring in recent years in Poland, providing evidence of reassortment with parallel persistence of particular gene combinations. Furthermore, relationship with notifiable poultry isolates emphasises the need for surveillance for AIV in wild birds and their genetic analysis.

Conflict of Interests Statement: The authors declare that there is no conflict of interests regarding the publication of this article.

Financial Disclosure Statement: The research was financially supported by the National Veterinary Research Institute in Pulawy, Poland.

Animal Rights Statement: None required.

Acknowledgements: We are grateful to Agnieszka Stolarek for creating the map.

\section{References}

1. Alexander D.J.: A review of avian influenza in different bird species. Vet Microbiol 2000, 74, 3-13.

2. Baek Y.H., Pascua P.N., Song M.S., Park K.J., Kwon H.I., Lee J.H., Kim S.Y., Moon H.J., Kim C.J., Choi Y.K.: Surveillance and characterization of low pathogenic H5 avian influenza viruses isolated from wild migratory birds in Korea. Virus Res 2010, 150, 119-128.

3. Bodewes R., Bestebroer T.M., van der Vries E., Verhagen J.H., Herfst S., Koopmans M.P., Fouchier R.A., Pfankuche V.M., Wohlsein P., Siebert U., Baumgärtner W., Osterhaus A.D.: Avian influenza $\mathrm{A}(\mathrm{H} 10 \mathrm{~N} 7)$ virus-associated mass deaths among harbor seals. Emerg Infect Dis 2015, 21, 720-722.

4. Briand F.X., Le Gall-Reculé G., Guillou-Cloarec C., Ogor K., Jestin V.: Phylogeny and genotyping of recent avian lowpathogenic H5 subtype influenza viruses from French ducks. J Gen Virol 2010, 91, 960-970.

5. Briand F.X., Schmitz A., Ogor K., Le Prioux A., GuillouCloarec C., Guillemoto C., Allée C., Le Bras M.O., Hirchaud E., Quenault H., Touzain F., Cherbonnel-Pansart M., Lemaitre E., Courtillon C., Gares H., Daniel P., Fediaevsky A., Massin P., Blanchard Y., Eterradossi N., van der Werf S., Jestin V., Niqueux E.: Emerging highly pathogenic H5 avian influenza viruses in France during winter 2015/16: phylogenetic analyses and markers for zoonotic potential. Euro Surveill 2017, 22, 30473.

6. Campitelli L., Mogavero E., De Marco M.A., Delogu M., Puzelli S., Frezza F., Facchini M., Chiapponi C., Foni E., Cordioli P., Webby R., Barigazzi G., Webster R.G., Donatelli I.: Interspecies transmission of an $\mathrm{H} 7 \mathrm{~N} 3$ influenza virus from wild birds to intensively reared domestic poultry in Italy. Virology 2004, 323, 24-36.
7. Cherbonnel M., Lamandé J., Allée C., Schmitz A., Ogor K., Le Gall-Reculé G., Le Bras M.O., Guillemoto C., Pierre I., Picault J.P., Jestin V.: Virologic findings in selected free-range mule duck farms at high risk for avian influenza infection. Avian Dis 2007, 51, 408-413.

8. Duan L., Campitelli L., Fan X.H., Leung Y.H., Vijaykrishna D., Zhang J.X., Donatelli I., Delogu M., Li K.S., Foni E., Chiapponi C., Wu W.L., Kai H., Webster R.G., Shortridge K.F., Peiris J.S., Smith G.J., Chen H., Guan Y.: Characterization of low-pathogenic H5 subtype influenza viruses from Eurasia: implications for the origin of highly pathogenic $\mathrm{H} 5 \mathrm{~N} 1$ viruses. J Virol 2007, 81, 7529-7539.

9. Global consortium for $\mathrm{H} 5 \mathrm{~N} 8$ and related influenza viruses. Role for migratory wild birds in the global spread of avian influenza H5N8. Science 2016, 354, 213-217.

10. LPAI confirmed in Hampshire. Vet Rec 2015, 176, 135. doi:10.1136/vr.h630.

11. Monne I., Fusaro A., Nelson M.I., Bonfanti L., Mulatti P., Hughes J., Murcia P.R., Schivo A., Valastro V., Moreno A., Holmes E.C., Cattoli G.: Emergence of a highly pathogenic avian influenza virus from a low-pathogenic progenitor. J Virol 2014, 88, 4375-4388.

12. Mughini-Gras L., Bonfanti L., Mulatti P., Monne I., Guberti V., Cordioli P., Marangon S.: Environmental correlates of H5N2 low pathogenicity avian influenza outbreak heterogeneity in domestic poultry in Italy. PLoS One 2014, 9, e86788.

13. Munster V.J., Wallensten A., Baas C., Rimmelzwaan G.F., Schutten M., Olsen B., Osterhaus A.D., Fouchier R.A.: Mallards and highly pathogenic avian influenza ancestral viruses, Northern Europe. Emerg Infect Dis 2005, 11, 1545-1551.

14. Munster V.J., Baas C., Lexmond P., Waldenstrom J., Wallensten A., Fransson T., Rimmelzwaan G.F., Beyer W.E., Schutten M., Olsen B., Osterhaus A.D., Fouchier R.A.: Spatial, temporal, and species variation in prevalence of influenza A viruses in wild migratory birds. PLoS Pathog 2007, 3, e61.

15. Nagy A., Cerníková L., Křivda V., Horníčková J.: Digital genotyping of avian influenza viruses of $\mathrm{H} 7$ subtype detected in central Europe in 2007-2011. Virus Res 2012, 165, 126-133.

16. Nagy A., Cerníková L., Jiřincová H., Havlíčková M., Horníčková J.: Local-scale diversity and between-year "frozen evolution" of avian influenza A viruses in nature. PLoS One 2014, 9, e103053.

17. Olsen B., Munster V.J., Wallensten A., Waldenstrom J., Osterhaus A.D., Fouchier R.A.: Global patterns of influenza A virus in wild birds. Science 2006, 312, 384-388.

18. Perdue M.L. Molecular determinants of pathogenicity for avian influenza viruses. In: Avian Influenza, edited by D.E. Swayne. Blackwell Publishing, Ames, 2008, pp. 23-41.

19. Pikuła A., Smietanka K., Lisowska A., Minta Z.: Active surveillance in poultry in Poland for avian influenza subtypes H5 and H7. Acta Biochim Pol 2014, 61, 459-463.

20. Scottish LPAI outbreak linked to wild birds. Vet Rec 2016, 178, 108. doi: 10.1136/vr.i487.

21. Snoeck C.J., Adeyanju A.T., De Landtsheer S., Ottosson U., Manu S., Hagemeijer W., Mundkur T., Muller C.P.: Reassortant low-pathogenic avian influenza H5N2 viruses in African wild birds. J Gen Virol 2011, 92, 1172-1183.

22. Stegeman A., Bouma A., Elbers A.R., de Jong M.C., Nodelijk G., de Klerk F., van Boven M.: Avian Influenza A virus (H7N7) epidemic in the Netherlands in 2003: course of the epidemic and effectiveness of control measures. J Infect Dis 2004, 190, 2088-2095.

23. Sultan S., Bui V.N., Hill N.J., Hussein I.T., Trinh D.Q., Inage K., Hashizume T., Runstadler J.A., Ogawa H., Imai K.: Genetic characterization of $\mathrm{H} 5 \mathrm{~N} 2$ influenza viruses isolated from wild birds in Japan suggests multiple reassortment. Arch Virol 2016, 161, 3309-3322.

24. Swayne D.E., Suarez D.L., Sims L.D.: Influenza. In: Diseases of poultry, edited by D.E. Swayne, Wiley-Blackwell, Ames, 2013, pp. 181-218. 
25. Śmietanka K., Minta Z.: Avian influenza in Poland. Acta Biochim Pol 2014, 61, 453-457.

26. Świętoń E., Wyrostek K., Jóźwiak M., Olszewska-Tomczyk M., Domańska-Blicharz K., Meissner W., Włodarczyk R., Minias P., Janiszewski T., Minta Z., Śmietanka K.: Surveillance for avian influenza virus in wild birds in Poland, 2008-15. J Wildl Dis 2017, 53, 330-338.

27. Tamura K., Stecher G., Peterson D., Filipski A., Kumar S.: MEGA6: molecular evolutionary genetics analysis version 6.0. Mol Biol Evol 2013, 30, 2725-2729.
28. Vasin A.V., Temkina O.A., Egorov V.V., Klotchenko S.A., Plotnikova M.A., Kiselev O.I.: Molecular mechanisms enhancing the proteome of influenza A viruses: an overview of recently discovered proteins. Virus Res 2014, 185, 53-63.

29. Wille M., Tolf C., Avril A., Latorre-Margalef N., Wallerström S., Olsen B., Waldenström J.: Frequency and patterns of reassortment in natural influenza A virus infection in a reservoir host. Virology 2013, 443, 150-160. 